\title{
GÉOMÉTRIE ALGÉBRIQUE PAR MORCEAUX, $K$-ÉQUIVALENCE ET MOTIFS
}

\author{
par Florian IVORRA et Julien SEBAG
}

RÉSUMÉ. Soit $k$ un corps algébriquement clos de caractéristique zéro. Deux $k$-variétés $K$-équivalentes sont-elles isomorphes par morceaux ? Ont-elles des motifs de Chow ou des groupes de Chow isomorphes? Dans cet article, nous relions toutes ces questions entre elles. Nous justifions les liens en question, en nous fondant sur certaines conjectures et certains résultats classiques de théorie des motifs, mais également en prouvant quelques énoncés non conjecturaux. Tous ces éléments fournissent les premiers indices de l'existence de telles relations.

\section{INTRODUCTION}

Dans cet article, $k$ est un corps. Une $k$-variété est un $k$-schéma de type fini, séparé. Une $k$-courbe est une $k$-variété purement de dimension 1 ; une $k$-surface est une $k$-variété purement de dimension 2.

0.1. On appelle variété de Calabi-Yau sur $k$ toute $k$-variété connexe, propre et lisse sur $k$, de diviseur canonique $K_{X}$ trivial. Cette classe de variétés algébriques se trouve profondément liée à des problèmes physiques et mathématiques. Dans [3] Batyrev a montré l'invariance birationnelle des nombres de Betti des variétés de Calabi-Yau complexes:

THÉORÈME ([3, Theorem 1.1]). Soient $X$ et $Y$ deux variétés complexes de Calabi-Yau, de dimension $n$. Si les variétés $X, Y$ sont birationnellement équivalentes, alors elles ont mêmes nombres de Betti, i.e.

$$
\operatorname{dim}_{\mathbf{C}} H^{i}(X, \mathbf{C})=\operatorname{dim}_{\mathbf{C}} H^{i}(Y, \mathbf{C}),
$$

pour tout $i, 0 \leqslant i \leqslant 2 n$. 
Ce résultat conduit à s'interroger sur la nature des propriétés géométriques que partagent deux variétés de Calabi-Yau birationnellement équivalentes, ou plus généralement deux $k$-variétés $K$-équivalentes.

0.2. Rappelons que deux $k$-variétés $X$ et $Y$ connexes, propres et lisses sur $k$, sont dites $K$-équivalentes s'il existe une $k$-variété $Z$ connexe, propre et lisse sur $k$, et des morphismes birationnels de $k$-schémas $X \stackrel{f}{\leftarrow} Z \stackrel{g}{\rightarrow} Y$ tels que les diviseurs canoniques relatifs $K_{Z / X}$ et $K_{Z / Y}$ soient égaux. Les couples de variétés de Calabi-Yau birationnellement équivalentes fournissent de tels exemples, et la $K$-équivalence a suscité de nombreux développements récents en géométrie birationnelle, notamment autour des motifs ou des catégories dérivées.

Le théorème de Batyrev peut-il se généraliser aux couples de $k$-variétés $K$-équivalentes? Une réponse positive à cette question peut se trouver dans [3, Theorem 4.2], et dans la généralisation suivante ${ }^{1}$ ), due à Kontsevich.

THÉORÈME ([19]). Soient $X$ et $Y$ deux variétés complexes connexes, propres et lisses sur $\mathbf{C}, K$-équivalentes, de dimension $n$. Alors les variétés $X$ et $Y$ ont mêmes nombres de Hodge, i.e.

$$
\operatorname{dim}_{\mathbf{C}} H^{q}\left(X, \Omega_{X \mid \mathbf{C}}^{p}\right)=\operatorname{dim}_{\mathbf{C}} H^{q}\left(Y, \Omega_{Y \mid \mathbf{C}}^{p}\right),
$$

pour tout $p, q, 0 \leqslant p, q \leqslant n$.

0.3. Les nombres de Betti ou de Hodge d'une $k$-variété, projective et lisse sur $k$, n'étant que des avatars numériques de son motif, il apparaît raisonnable de se demander si l'invariance des nombres de Hodge au sein d'une même classe de $K$-équivalence ne serait pas simplement le reflet d'un résultat de nature motivique.

QUESTION. Deux k-variétés connexes, projectives et lisses sur $k, K$-equivalentes, ont-elles des motifs de Chow isomorphes, ou, au moins, des groupes de Chow isomorphes?

Une réponse positive à cette question difficile a été donnée dans un certain nombre de cas très spécifiques de $K$-équivalence, comme le flop ordinaire

\footnotetext{
${ }^{1}$ ) Ce théorème joue historiquement un rôle important, car il est à l'origine du développement de la théorie géométrique de l'intégration motivique. Introduite par Kontsevich dans [19], l'intégration motivique a permis de fournir une première preuve de cette généralisation du théorème de Batyrev.
} 
(cf. [22, Theorem 0.1], ou [28, Theorem 1] pour une preuve radicalement différente).

0.4. Dans le présent travail, nous nous intéressons à la question ci-dessus, et particulièrement aux liens qu'elle entretient avec ce que l'on appellera la géométrie algébrique par morceaux, point de vue «introduit» par Grothendieck dans ÉGA (voir [11, 7.2.14]), ou dans une lettre à J.-P. Serre écrite ${ }^{2}$ ) en 1964.

Dans ce travail, nos motivations sont doubles. Premièrement, nous souhaitons justifier et clarifier ce lien avec la géométrie par morceaux. Pour cela nous relions entre elles différentes questions ouvertes, qui soit n'apparaissent pas dans la littérature, soit s'y trouvent dispersées dans différents domaines. Dans ce tableau, il nous semble, de manière peut-être surprenante, que l'anneau de Grothendieck des variétés (voir section 1) et la géométrie par morceaux (voir section 2) pourraient jouer un rôle important dans la compréhension de ces différents problèmes liant $K$-équivalence et motifs (voir section 3 ). Ainsi, dans le cas de la question énoncée au paragraphe 0.3 , la géométrie algébrique par morceaux suggère le «dévissage» suivant:

ÉTAPE 1. Deux $k$-variétés connexes, projectives et lisses sur $k, K$-équivalentes sont-elles isomorphes par morceaux, ou ont-elles même classe dans l'anneau de Grothendieck des variétés?

ÉTAPE 2. Deux k-variétés connexes, projectives et lisses sur $k$, qui ont la même classe dans l'anneau de Grothendieck des variétés, ont-elles des motifs de Chow isomorphes, ou des groupes de Chow isomorphes?

$\mathrm{Au}$ paragraphe 4.1, nous expliquons comment la deuxième étape est une conséquence directe de la conjecture de dimension finie de Kimura [17] et O'Sullivan, via le théorème de nilpotence de Kimura ${ }^{3}$ ) et du théorème de semisimplicité de Jannsen. Les étapes 1 et 2 soulignent la nature "par morceaux », a priori non apparente, de la question énoncée au paragraphe 0.3 , et fournissent par ce biais un point de vue original susceptible d'aider à la compréhension du problème.

\footnotetext{
2) Dans cette même lettre du 16 août 1964, Grothendieck introduisait la notion de motifs de Chow.

3 ) Ce théorème est démontré pour les $k$-variétés, projectives et lisses sur le corps $k$, dont le motif de Chow associé est de dimension finie au sens de [17].
} 
0.5. Deuxièmement, nous donnons quelques résultats positifs et non conjecturaux aux questions énoncées à la section 3. Ces énoncés simples, traitant essentiellement des cas de petites dimensions, constituent toutefois des indices non triviaux laissant espérer que ces questions puissent admettre une réponse positive en général. Nous montrons en particulier que la question de l'étape 2 admet une réponse positive pour les surfaces projectives et lisses sur $k$ (voir proposition 4.6). La proposition 5.5 fournit de nouveaux cas pour lesquels la question de l'étape 1 possède une réponse positive.

0.6. Enfin, la notion d'isomorphisme par morceaux est cruciale dans les différents développements de ce travail. Nous l'étudions succinctement au paragraphe 2 .

\section{PRÉLIMINAIRES}

1.1. Soit $\mathbf{Z}\left[\operatorname{Var}_{k}\right]$ le groupe abélien libre engendré par les classes d'isomorphisme des $k$-variétés. On note $\{X\}$ la classe de la $k$-variété $X$ dans $\mathbf{Z}\left[\operatorname{Var}_{k}\right]$. L'anneau de Grothendieck des variétés est le groupe abélien obtenu comme le quotient de $\mathbf{Z}\left[\operatorname{Var}_{k}\right]$ par le sous-groupe engendré par les éléments de $\mathbf{Z}\left[\operatorname{Var}_{k}\right]$ de la forme $\{X\}-\{Z\}-\{X \backslash Z\}$, où $X$ est une $k$-variété et $Z$ un sous-schéma fermé de $X$. On le note $K_{0}\left(\operatorname{Var}_{k}\right)$; les relations ci-dessus sont appelées relations de découpage. Dans l'anneau $K_{0}\left(\operatorname{Var}_{k}\right)$, on notera $[X]$ la classe de la variété $X$. Le produit fibré au-dessus de $k$ induit une structure d'anneau sur le groupe $K_{0}\left(\operatorname{Var}_{k}\right)$ en posant $[X] \cdot[Y]:=\left[X \times_{k} Y\right]$ pour toutes $k$-variétés $X$ et $Y$. Il est clair que l'élément neutre de cette structure est $1=[\operatorname{Spec}(k)]$. On note $\mathbf{L}:=\left[\mathbf{A}_{k}^{1}\right]$ la classe de la droite affine $\mathbf{A}_{k}^{1}$ dans l'anneau $K_{0}\left(\operatorname{Var}_{k}\right)$ et $\mathscr{M}_{k}$ la localisation de l'anneau $K_{0}\left(\operatorname{Var}_{k}\right)$ par L, i.e.

$$
\mathscr{M}_{k}:=K_{0}\left(\operatorname{Var}_{k}\right)\left[\mathbf{L}^{-1}\right]
$$

1.2. La théorie de l'intégration motivique, introduite dans sa version géométrique par Kontsevich, définit des intégrales qui prennent leurs valeurs dans une complétion $\widehat{\mathscr{M}_{k}}$ de l'anneau $\mathscr{M}_{k}$, construite à partir d'une filtration naturellement associée à la dimension (voir [30, §4.1] pour une définition 
précise de cette filtration). Grâce à cette théorie ${ }^{4}$ ), Kontsevich a établi le théorème suivant (dont l'énoncé généralise la version de l'introduction).

THÉORÈME 1.1 (Kontsevich). Soit $k$ un corps de caractéristique zéro. Soient $X$ et $Y$ deux $k$-variétés connexes, propres et lisses sur $k$, et $K$-équivalentes ${ }^{5}$ ). Alors $[X]=[Y]$ dans $\widehat{\mathscr{M}}_{k}$.

1.3. Soit $k$ un corps de caractéristique zéro. Soit $X$ une $k$-variété connexe, propre et lisse sur $k$. L'on définit son polynôme de Poincaré par

$$
P_{X}(u)=\sum b_{n}(X) u^{n} \in \mathbf{Z}[u]
$$

avec $b_{n}(X):=\operatorname{dim}_{k} H_{\mathrm{dR}}^{n}(X)$. Si $k$ est un sous-corps de $\mathbf{C}$, l'on définit son polynôme de Hodge par

$$
H_{X}(u, v):=\sum h^{p, q}(X) u^{p} v^{q} \in \mathbf{Z}[u, v],
$$

où $h^{p, q}(X):=\operatorname{dim}_{k} H^{q}\left(X, \Omega_{X}^{p}\right)$. Notons que le théorème de décomposition de Hodge fournit, dans ce cas, la formule $H_{X}(u, u)=P_{X}(u)$. En outre, les relations de découpage dans l'anneau $K_{0}\left(\operatorname{Var}_{k}\right)$ permettent d'étendre ces définitions en la donnée de morphismes d'anneaux

$$
P: \mathscr{M}_{k} \rightarrow \mathbf{Z}\left[u, u^{-1}\right] \quad \text { et } H: \mathscr{M}_{k} \rightarrow \mathbf{Z}\left[u, v,(u v)^{-1}\right] .
$$

En évaluant $P$ en -1 , l'on obtient la caractéristique d'Euler $\chi: \mathscr{M}_{k} \rightarrow \mathbf{Z}$.

REMARQuE 1.2. Soit $k$ un corps de caractéristique zéro. Soient $W$ une $k$-variété de dimension $d$, et $C_{1}, \ldots, C_{n}$ ses composantes irréductibles de dimension $d$. Par le théorème de Hironaka sur la résolution des singularités, le lemme de Chow, et la relation $[X]=\left[X_{\text {red }}\right]$ dans $K_{0}\left(\operatorname{Var}_{k}\right)$, il existe, pour toute $k$-variété $X$, des $k$-variétés $D_{1}, \ldots, D_{n}$, connexes, projectives et lisses sur $k$, birationnellement équivalentes à $\left(C_{1}\right)_{\text {red }}, \ldots,\left(C_{n}\right)_{\text {red }}$ respectivement, et des $k$-variétés $E_{1}, \ldots, E_{m}$ connexes, projectives et lisses sur $k$, de dimension au plus $d-1$, telles que

$$
[W]=\sum_{i=1}^{n}\left[D_{i}\right]+\sum_{j=1}^{m} \varepsilon_{j}\left[E_{j}\right],
$$

dans l'anneau $K_{0}\left(\operatorname{Var}_{k}\right)$, avec $\varepsilon_{j}= \pm 1$.

\footnotetext{
${ }^{4}$ ) Grâce à l'existence des factorisations faibles des applications birationnelles, le théorème suivant peut s'obtenir aujourd'hui sans utiliser l'intégration motivique, et sans passer à la complétion de $K_{0}\left(\operatorname{Var}_{k}\right)$, cf. [33, 7.10] ou [5, Remark 1.2].

${ }^{5}$ ) Pour la définition, voir 0.2 .
} 
Comme le polynôme de Poincaré d'une $k$-variété $X$ connexe, propre et lisse sur $k$, possède un degré égal à $2 \operatorname{dim}(X)$, et a un coefficient dominant égal au nombre de composantes irréductibles géométriques (i.e. de $X_{\bar{k}}$ avec $\bar{k}$ une clôture algébrique de $k$ ) de dimension maximale, l'on voit facilement, par la relation (1.1), que deux $k$-variétés $X$ et $Y$ telles que $P([X])=P([Y])$ ont la même dimension et le même nombre de composantes irréductibles géométriques de dimension maximale.

Notons que les morphismes d'anneaux $H$ et $P$ se factorisent par l'image de $\mathscr{M}_{k}$ dans $\widehat{\mathscr{M}}_{k}$ (cf. [5, Preuve du théorème 6.1.1]). Le théorème 1.1 assure donc en particulier que deux $k$-variétés connexes, propres et lisses sur $k, K$-équivalentes ont le même polynôme de Poincaré. L'on déduit de la remarque précédente l'important corollaire suivant.

COROLlaIRE 1.3. Soit $k$ un corps algébriquement clos de caractéristique zéro. Soient $X$ et $Y$ deux $k$-variétés connexes, propres et lisses sur $k$, $K$-équivalentes de dimension $d$. Soient $F_{X}$ et $F_{Y}$ deux $k$-variétés réduites telles que $[X]-\left[F_{X}\right]=[Y]-\left[F_{Y}\right]$ dans l'anneau $K_{0}\left(\operatorname{Var}_{k}\right)$. Alors les $k$-variétés $F_{X}$ et $F_{Y}$ ont la même dimension et le même nombre de composantes irréductibles de dimension maximale.

Démonstration. Par hypothèse, $[X]-\left[F_{X}\right]=[Y]-\left[F_{Y}\right]$ dans l'anneau $K_{0}\left(\operatorname{Var}_{k}\right)$ et donc $[X]=[Y]$ dans sa complétion $\widehat{\mathscr{M}_{k}}$. En vertu du théorème 1.1, l'on conclut $\left[F_{X}\right]=\left[F_{Y}\right]$ dans l'anneau $\widehat{\mathscr{M}_{k}}$. Par conséquent, les $k$-variétés $F_{X}$ et $F_{Y}$ ont le même polynôme de Poincaré. Le résulat découle alors de la remarque 1.2 .

1.4. Rappelons que deux $k$-variétés réduites $X$ et $Y$ sont dites stablement birationnelles s'il existe deux entiers $m, \ell \in \mathbf{N}$ tels que $X \times{ }_{k} \mathbf{P}_{k}^{m}$ et $Y \times{ }_{k} \mathbf{P}_{k}^{\ell}$ soient birationnellement équivalentes.

Désignons par $\mathbf{Z}[S B]$ le groupe abélien libre engendré par les classes d'équivalence des $k$-variétés connexes, propres et lisses sur $k$, pour la relation «d'être stablement birationnel» (appelée relation d'équivalence stable). Larsen et Lunts ont prouvé le résultat suivant.

THÉORÈME 1.4 ([21], voir également [4]). Soit $k$ un corps algébriquement clos de caractéristique zéro. Il existe un unique morphisme d'anneaux

$$
\mathrm{SB}: K_{0}\left(\operatorname{Var}_{k}\right) \rightarrow \mathbf{Z}[S B]
$$


qui envoie la classe d'une $k$-variété connexe, propre et lisse sur $k$, sur sa classe d'équivalence par la relation d'équivalence stable. Ce morphisme est surjectif et son noyau est l'idéal de $K_{0}\left(\operatorname{Var}_{k}\right)$ engendré par $\mathbf{L}$.

\section{LES ISOMORPHISMES PAR MORCEAUX}

2.1. Rappelons ${ }^{6}$ ) que deux $k$-schémas de type fini $X$ et $Y$ sont dits isomorphes par morceaux s'il existe une partition $\left(X_{i}\right)_{0 \leqslant i \leqslant n}\left(\operatorname{resp} .\left(Y_{i}\right)_{0 \leqslant i \leqslant n}\right)$ de $X$ (resp. de $Y$ ) en sous-schémas localement fermés, telles que, pour tout $i$, $0 \leqslant i \leqslant n$, les $k$-schémas $\left(X_{i}\right)_{\text {red }}$ et $\left(Y_{i}\right)_{\text {red }}$ soient isomorphes. Par définition, la donnée de deux $k$-variétés isomorphes par morceaux induit donc celle d'une collection d'isomorphismes de $k$-schémas $\left(f_{0}, \ldots, f_{n}\right)$ tels que $f_{i}$ identifie $\left(X_{i}\right)_{\text {red }}$ et $\left(Y_{i}\right)_{\text {red }}$, pour tout $i, 0 \leqslant i \leqslant n$. La donnée d'une telle famille $\left(f_{0}, \ldots, f_{n}\right)$ sera appelée isomorphisme par morceaux. Notons enfin que la donnée d'un isomorphisme par morceaux diffère grandement de celle d'un isomorphisme de $k$-schémas. Pour illustrer ce point, notons que les $k$-variétés suivantes sont deux à deux isomorphes par morceaux (et non isomorphes en tant que $k$-schémas): $\mathbf{A}_{k}^{1}, \operatorname{Spec}\left(k[x, y] /\left(x^{3}-y^{2}\right)\right)$ et $\mathbf{G}_{m, k} \sqcup \operatorname{Spec}(k)$.

2.2. Les isomorphismes par morceaux abondent en géométrie algébrique. Nous en donnons ici quelques exemples.

EXEMPLE 2.1. a) La géométrie birationnelle, et particulièrement le programme de Mori, fournit de nombreux exemples d'isomorphismes par morceaux. Par exemple, les flops ordinaires, les flops de Mukai, les flops de Mukai stratifiés (et leurs composés) sont des isomorphismes par morceaux (cf. [7] pour les définitions).

b) Soit $k$ un corps de caractéristique zéro, de clôture algébrique $\bar{k}$. Soient $X$ et $Y$ deux $k$-variétés, et $f: X \rightarrow Y$ un morphisme de $k$-schémas. Si l'application d'ensembles $f(\bar{k}): X(\bar{k}) \rightarrow Y(\bar{k})$, induite par le morphisme $f$, est bijective, alors le morphisme $f$ fournit un isomorphisme par morceaux. C'est une conséquence du théorème principal de Zariski.

\footnotetext{
${ }^{6}$ ) En suivant [23], un point de vue équivalent, mais plus intrinsèque, peut être adopté. Ce point de vue a été introduit par Grothendieck dans [11, 7.2.14], puis sommairement développé dans $[26,27]$.
} 
2.3. Les relations de découpage dans l'anneau $K_{0}\left(\operatorname{Var}_{k}\right)$ assurent que deux $k$-variétés, isomorphes par morceaux, ont la même image dans l'anneau $K_{0}\left(\operatorname{Var}_{k}\right)$. Dans [21], Larsen et Lunts s'interrogent sur la validité de la réciproque. Ce problème, difficile, nous apparaît comme la pierre angulaire de la compréhension géométrique de l'anneau de Grothendieck des variétés. Formulons cette question.

Question LL. Soient $X$ et $Y$ deux $k$-variétés telles que $[X]=[Y]$ dans l'anneau $K_{0}\left(\operatorname{Var}_{k}\right)$. Les $k$-variétés $X$ et $Y$ sont-elles isomorphes par morceaux?

A l'heure actuelle, cette question est essentiellement ouverte. Si $k$ est un corps algébriquement clos de caractéristique zéro, des éléments de réponse peuvent être trouvés dans [23, 31]. La question suivante nous apparaît comme une étape importante dans toute tentative de réponse à la question LL.

QUESTION RAT. Soit $k$ un corps algébriquement clos de caractéristique zéro. Soit $X$ une $k$-variété, projective et lisse sur $k$, de dimension $d \geqslant 3$, stablement rationnelle et non rationnelle. La $k$-variété $X$ peut-elle avoir la classe d'une variété rationnelle dans l'anneau $K_{0}\left(\operatorname{Var}_{k}\right)$ ?

A l'aide de la question LL, nous pouvons reformuler et préciser comme suit une question posée par Gromov dans [10, 3.G'", p. 121].

QUESTION G. Soit k un corps algébriquement clos de caractéristique zéro. Soient $X$ une $k$-variété et $f: X \rightarrow X$ une application birationnelle, induisant un isomorphisme de $k$-schémas entre deux sous-variétés $U, V$ ouvertes et denses de $X$. Notons $Z_{U}$ et $Z_{V}$ les uniques sous-variétés fermées de $X$, réduites, de supports respectifs $X \backslash U$ et $X \backslash V$. Les $k$-variétés $Z_{U}$ et $Z_{V}$ sontelles isomorphes par morceaux?

Il est clair que, si la question LL possède une réponse positive, il en va de même de la question G. Les résultats de [31] permettent donc d'y répondre positivement dans le cas des courbes, des surfaces projectives et lisses sur $k$, et des $k$-variétés de dimension $d \geqslant 2$ n'ayant qu'un nombre fini de courbes rationnelles. Plus récemment, dans [20], Lamy et le second auteur ont répondu positivement à la question $\mathrm{G}$, dans le cas d'une variété complexe $X$, projective et lisse, de dimension 3 telle que $H^{0}\left(X, \Omega_{X \mid \mathbf{C}}^{3}\right)=H^{0}\left(X, \Omega_{X \mid \mathbf{C}}^{1}\right)=0$. 
2.4. Nous prouvons maintenant un critère original et simple pour déterminer si deux $k$-variétés de petite dimension sont isomorphes par morceaux. Soient $k$ un corps de caractéristique zéro, $X$ et $Y$ deux $k$-variétés de dimension $d \geqslant 1$.

Supposons que $X$ et $Y$ soient deux $k$-variétés isomorphes par morceaux. Alors il découle de la définition que l'ensemble $\Gamma_{X}$ des composantes irréductibles de dimension $d$ de $X_{\text {red }}$ est en bijection avec l'ensemble $\Gamma_{Y}$ des composantes irréductibles de dimension $d$ de $Y_{\text {red }}$. De plus, il existe une bijection $\sigma: \Gamma_{X} \rightarrow \Gamma_{Y}$ telle que les $k$-variétés $C$ et $\sigma(C)$ soient birationnellement équivalentes, pour tout $C \in \Gamma_{X}$. Enfin, $P([X])=P([Y])$ et $\chi([X])=\chi([Y])$, puisque $[X]=[Y]$ dans l'anneau $K_{0}\left(\operatorname{Var}_{k}\right)$.

Lorsque $d \in\{0,1,2\}$ la condition nécessaire ci-dessus devient un critère nécessaire et suffisant à l'existence d'un isomorphisme par morceaux.

PROPOSITION 2.2. Soit $k$ un corps algébriquement clos de caractéristique zéro. Supposons que la dimension de $X$ soit au plus 1 . Les $k$-variétés $X$ et $Y$ sont isomorphes par morceaux si et seulement si les deux assertions suivantes sont vérifiées.

(1) Il existe une bijection $\sigma: \Gamma_{X} \rightarrow \Gamma_{Y}$ telle que les $k$-variétés $C$ et $\sigma(C)$ soient birationnellement équivalentes, pour tout $C \in \Gamma_{X}$;

(2) $\chi([X])=\chi([Y])$.

Démonstration. Supposons vérifiées les conditions (1) et (2). Ou bien la dimension de $X$ est égale à 0 et dans ce cas le point (2) conclut directement; ou bien les $k$-variétés sont de dimension 1 . Dans ce dernier cas, il existe, en vertu du point (1), un sous-schéma ouvert $U$ de $X_{\text {red }}$ et un sous-schéma ouvert $V$ de $Y_{\text {red }}$ tels que $U$ et $V$ soient $k$-isomorphes, et tels que les sous-variétés fermées $(X \backslash U)_{\text {red }}$ et $(Y \backslash V)_{\text {red }}$ soient de dimension 0, i.e. des sommes disjointes de points rationnels. Posons $(X \backslash U)_{\mathrm{red}}=\sqcup_{i=1}^{r} \operatorname{Spec}(k)$ et $(Y \backslash V)_{\text {red }}=\sqcup_{i=1}^{s} \operatorname{Spec}(k)$. Par les relations de découpage, l'on obtient

$$
[X]-r=[U]=[V]=[Y]-s
$$

dans l'anneau $K_{0}\left(\operatorname{Var}_{k}\right)$. D'où

$$
\chi([X])-r=\chi([Y])-s \quad \text { et } \quad r=s .
$$

Le $k$-isomorphisme $f_{0}: U \rightarrow V$ se complète alors facilement en un isomorphisme par morceaux, via tout isomorphisme de $k$-schémas $f_{1}: \sqcup_{i=1}^{r} \operatorname{Spec}(k) \rightarrow$ $\sqcup_{i=1}^{s} \operatorname{Spec}(k)$. 
PROPOSITION 2.3. Soit $k$ un corps algébriquement clos de caractéristique zéro. Supposons que les $k$-variétés $X$ et $Y$ soient de dimension au plus 2, propres et lisses sur $k$. Alors les $k$-variétés $X$ et $Y$ sont isomorphes par morceaux si et seulement si les deux assertions suivantes sont vérifiées.

(1) Il existe une bijection $\sigma: \Gamma_{X} \rightarrow \Gamma_{Y}$ telle que les $k$-variétés $C$ et $\sigma(C)$ soient birationnellement équivalentes, pour tout $C \in \Gamma_{X}$;

(2) $P([X])=P([Y])$.

Démonstration. Supposons vérifiées les conditions (1) et (2). Si la dimension de $X$ est au plus 1, l'on conclut par exemple en invoquant la proposition 2.2. Supposons désormais que les $k$-variétés $X$ et $Y$ sont de dimension 2. En vertu du point (1), il existe des sous-variétés fermées et réduites $C_{X}$ et $C_{Y}$ de $X$ et $Y$ de dimension au plus 1, et un isomorphisme de $k$-schémas $f_{0}: X \backslash C_{X} \rightarrow Y \backslash C_{Y}$. Par les relations de découpage, on a:

$$
[X]-\left[C_{X}\right]=[Y]-\left[C_{Y}\right]
$$

dans l'anneau $K_{0}\left(\operatorname{Var}_{k}\right)$. En particulier, $P([X])-P\left(\left[C_{X}\right]\right)=P([Y])-P\left(\left[C_{Y}\right]\right)$ dans l'anneau de polynômes $\mathbf{Z}[u]$. Il en découle du point (2) que:

$$
P\left(\left[C_{X}\right]\right)=P\left(\left[C_{Y}\right]\right)
$$

dans $\mathbf{Z}[u]$. En vertu de la remarque 1.2, l'on déduit que les $k$-variétés $C_{X}$ et $C_{Y}$ ont donc la même dimension et le même nombre de composantes irréductibles de dimension maximale. Si cette dimension était 0 , nous déduirions automatiquement que les $k$-variétés $C_{X}$ et $C_{Y}$ sont isomorphes, et donc que les $k$-variétés $X$ et $Y$ sont isomorphes par morceaux. Supposons que la dimension de $C_{X}$ est égale à 1 . Soient $D_{X, i}, D_{Y, i}$, pour $1 \leqslant i \leqslant m$, les modèles projectifs et lisses sur $k$ des composantes irréductibles de dimension 1 de $C_{X}$ et $C_{Y}$ respectivement. Grâce aux relations de découpage, il existe un entier $n \in \mathbf{Z}$ tel que

$$
[X]-[Y]=\sum_{i=1}^{m}\left[D_{X, i}\right]-\sum_{i=1}^{m}\left[D_{Y, i}\right]+n
$$

dans l'anneau $K_{0}\left(\operatorname{Var}_{k}\right)$. On peut alors appliquer le morphisme SB à cette relation (cf. théorème 1.4), ce qui donne

$$
\sum_{i=1}^{m} \mathrm{SB}\left(\left[D_{X, i}\right]\right)-\sum_{i=1}^{m} \mathrm{SB}\left(\left[D_{Y, i}\right]\right)=-n
$$

dans le groupe abélien libre $\mathbf{Z}[S B]$. On en conclut que $n=0$, et que, quitte à renuméroter, $D_{X, i}$ est $k$-isomorphe à $D_{Y, i}$, pour tout $i, 1 \leqslant i \leqslant m$. Autrement 
dit, les composantes irréductibles des $k$-variétés $C_{X}$ et $C_{Y}$ correspondantes sont birationnellement équivalentes. Les $k$-variétés $C_{X}$ et $C_{Y}$ ont en outre la même caractéristique d'Euler. Donc, par la proposition 2.2, elles sont isomorphes par morceaux. Soit $\left(f_{1}, f_{2}\right)$ un isomorphisme par morceaux entre $C_{X}$ et $C_{Y}$. Alors $\left(f_{0}, f_{1}, f_{2}\right)$ est un isomorphisme par morceaux entre $X$ et $Y$.

\section{PANORAMA DE PROBLÈMES DE GÉOMÉTRIE ALGÉBRIQUE PAR MORCEAUX}

\subsection{UNE LISTE DE QUESTIONS EN GÉOMÉTRIE ALGÉBRIQUE PAR MORCEAUX}

Dans cette section, nous explicitons les questions qui forment le cœur du présent article. Les problèmes sous-jacents restent essentiellement ouverts à l'heure actuelle. Nous renvoyons également au paragraphe 3.2 pour une synthèse des relations qui existent entre ces différents problèmes. Nous supposons dans cette section que la caractéristique du corps $k$ est zéro.

3.1.1. Comme nous l'avons souligné dans l'introduction, les théorèmes de Batyrev et Kontsevich sur la $K$-équivalence incitent à poser la question plus générale suivante, reliant $K$-équivalence et motifs.

QUESTION KM. Deux k-variétés $X$ et $Y$ connexes, projectives et lisses sur $k$, et $K$-équivalentes ont-elles des motifs de Chow $h_{\text {rat }}(X)$ et $h_{\text {rat }}(Y)$ isomorphes dans $\mathrm{M}_{\mathrm{rat}}(k ; \mathbf{Q})$ ?

Cette question peut se trouver dans la littérature (voir, par exemple, [34] pour un énoncé légèrement affiné) et possède la version plus faible suivante.

QUESTION KC. Deux k-variétés $X$ et $Y$ connexes, projectives et lisses sur $k$ et $K$-équivalentes ont-elles des groupes de Chow $\mathrm{CH}^{n}(X)_{\mathbf{Q}}$ et $\mathrm{CH}^{n}(Y)_{\mathbf{Q}}$ isomorphes?

Dans le reste de cette section, nous proposons un «dévissage» en deux étapes de la question $K M$ (resp. $K C$ ), en les reliant aux questions KP et PM (resp. KP et PC), qui semblent exhiber la nature «par morceaux», a priori non apparente, de la question KM (resp. KC).

3.1.2. La première question relie la relation de $K$-équivalence à la géométrie algébrique par morceaux. Elle s'énonce comme suit: 
QUESTIONS KP. Soient $X$ et $Y$ deux $k$-variétés connexes, projectives et lisses, $K$-équivalentes.

$\mathrm{KP}$ : Les $k$-variétés $X$ et $Y$ sont-elles isomorphes par morceaux?

Cette question admet la formulation a priori plus faible suivante.

$\mathrm{KPw}$ : Les $k$-variétés $X$ et $Y$ ont-elles la même classe dans l'anneau $K_{0}\left(\operatorname{Var}_{k}\right)$ ?

La question KPw a été initialement posée par Yasuda. Notons également qu'une réponse positive à la question KP fournirait un résultat $a$ priori plus fin que le théorème de Kontsevich (cf. théorème 1.1). En effet, d'une part, le théorème 1.1 n'affirme que l'égalité des classes dans l'anneau $\widehat{\mathscr{M}_{k}}$ (ou dans une localisation de l'anneau $K_{0}\left(\operatorname{Var}_{k}\right)$, comme le souligne notre première note de bas de page), et, d'autre part, l'égalité de classes dans l'anneau $K_{0}\left(\operatorname{Var}_{k}\right)$ ne garantit pas, à l'heure actuelle en tout cas, l'existence d'un isomorphisme par morceaux entre les variétés considérées. L'obstruction pour répondre à la question $\mathrm{KPw}$ directement à partir du théorème de Kontsevich réside dans les deux questions suivantes, qui sont, quant à elles, totalement ouvertes à l'heure d'aujourd'hui.

- Le morphisme de complétion $\mathscr{M}_{k} \rightarrow \widehat{\mathscr{M}_{k}}$ est-il injectif?

- Le morphisme de localisation $K_{0}\left(\operatorname{Var}_{k}\right) \rightarrow \mathscr{M}_{k}$ est-il injectif?

Remarque 3.1. a) Dans [31], le second auteur a relié la question de l'injectivité du morphisme $K_{0}\left(\operatorname{Var}_{k}\right) \rightarrow \mathscr{M}_{k}$ à la question LL.

b) Différents problèmes de simplification «à la Zariski» constituent a priori des obstructions à la bonne compréhension de la question KPw (voir l'assertion a) ou le théorème 5.4). Ce type de problèmes semble pouvoir être formellement contourné en remplaçant l'existence d'un isomorphisme par morceaux (resp. l'égalité des classes dans l'anneau $K_{0}\left(\operatorname{Var}_{k}\right)$ ) dans la formulation de la question KP (resp. KPw) par l'égalité des classes dans l'anneau $\mathscr{M}_{k}=K_{0}\left(\operatorname{Var}_{k}\right)\left[\mathbf{L}^{-1}\right]$.

En général, l'affaiblissement de la formulation consistant à passer à la localisation n'est pas triviale. Nous renvoyons le lecteur à [15], où est illustré ce phénomène dans l'étude de la fonction zêta de Kapranov.

3.1.3. La seconde question relie l'existence d'isomorphismes par morceaux aux motifs et groupes de Chow, de la manière précise suivante.

QUESTIONS PM. Soient $X$ et $Y$ deux $k$-variétés projectives et lisses sur $k$.

PM : Deux $k$-variétés $X$ et $Y$ sont isomorphes par morceaux ont-elles des motifs de Chow $h_{\text {rat }}(X)$ et $h_{\text {rat }}(Y)$ isomorphes dans $\mathrm{M}_{\text {rat }}(k ; \mathbf{Q})$ ? 
Cette question possède la formulation a priori plus forte suivante.

PMs: Deux $k$-variétés $X$ et $Y$ qui ont la même classe dans l'anneau $K_{0}\left(\operatorname{Var}_{k}\right)$ ont-elles des motifs de Chow $h_{\text {rat }}(X)$ et $h_{\text {rat }}(Y)$ isomorphes dans $\mathrm{M}_{\mathrm{rat}}(k ; \mathbf{Q})$ ?

Comme, pour une $k$-variété $X$ projective et lisse sur $k, \mathrm{CH}^{n}(X)_{\mathbf{Q}}=$ $\operatorname{Hom}_{\mathrm{M}_{\text {rat }}(k ; \mathbf{Q})}\left(\mathbf{L}^{n}, h_{\text {rat }}(X)\right)$, les questions PM et PMs peuvent se formuler au niveau des groupes de Chow de la manière suivante.

QUESTIONS PC. Soient $X$ et $Y$ deux $k$-variétés projectives et lisses sur $k$. Soit $n$ un entier naturel.

$\mathrm{PC}^{n}$ : Deux $k$-variétés $X$ et $Y$ sont isomorphes par morceaux ont-elles des groupes de Chow $\mathrm{CH}^{n}(X)_{\mathbf{Q}}$ et $\mathrm{CH}^{n}(Y)_{\mathbf{Q}}$ isomorphes?

Cette question possède la formulation a priori plus forte suivante.

$\mathrm{PCs}^{n}$ : Deux $k$-variétés $X$ et $Y$ qui ont la même classe dans l'anneau $K_{0}\left(\operatorname{Var}_{k}\right)$ ont-elles des groupes de Chow $\mathrm{CH}^{n}(X)_{\mathbf{Q}}$ et $\mathrm{CH}^{n}(Y)_{\mathbf{Q}}$ isomorphes?

Remarque 3.2. Ces questions $P C$ admettent des réponses négatives si les $k$-variétés $X$ et $Y$ ne sont pas supposées, au minimum, propres sur $k$. Soient $X$ une $k$-variété réduite et $U$ une sous-variété ouverte (non vide). Si $Z$ est l'unique sous-variété fermée de $X$, réduite, de support égal à $X \backslash Z$. Alors les $k$-variétés $X$ et $U \sqcup Z$ sont isomorphes par morceaux, mais $\mathrm{CH}^{n}(X)_{\mathbf{Q}}$ n'est pas isomorphe à $\mathrm{CH}^{n}(U \sqcup Z)_{\mathbf{Q}}$.

EXEMPLE 3.3. Si $X$ et $X^{+}$sont deux variétés complexes, projectives et lisses sur $k$, liées par un flop ordinaire, alors $X$ et $X^{+}$sont isomorphes par morceaux (cf. exemple 2.1). Par ailleurs, dans [22, Theorem 01] (voir également [28, Theorem 1] pour une preuve totalement différente), il est prouvé que les motifs de Chow de $X$ et $X^{+}$sont isomorphes.

\subsection{UNE SYNTHÈSE DES DIFFÉRENTES QUESTIONS}

Le diagramme ci-dessous positionne les différentes propriétés autour desquelles s'articule notre discussion. Les fèches en pointillés correspondent aux questions que nous avons évoquées au paragraphe précédent (le symbole LL correpond à la question LL de la section 2, le symbole KS désigne la conjecture de Kimura et O'Sullivan, évoquée au paragraphe 4.1). Les doubles flèches représentent des implications inconditionnelles. Dans le diagramme $X$ et $Y$ sont deux $k$-variétés connexes, projectives et lisses sur $k$; on 
note $K_{0}\left(\mathrm{M}_{\text {rat }}\right)$ l'anneau $K_{0}\left(\mathrm{M}_{\text {rat }}(k ; \mathbf{Q})\right)$ et on appelle motif de $X$ l'élément $h_{\text {rat }}(X) \in \mathrm{M}_{\text {rat }}(k ; \mathbf{Q})$.

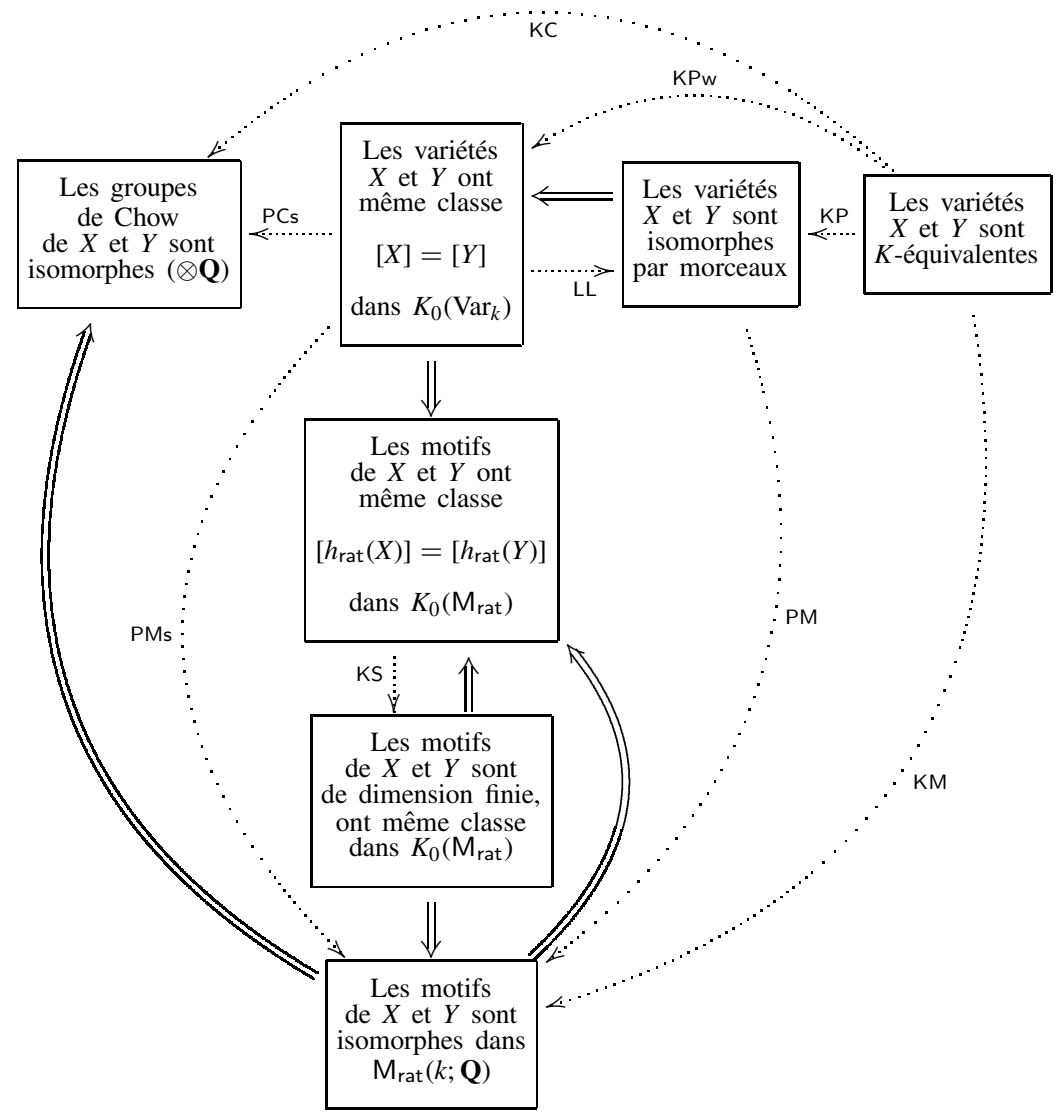

\section{GÉOMÉTRIE ALGÉBRIQUE PAR MORCEAUX ET MOTIFS}

\subsection{LA MOTIVATION DERRIÈRE PM}

4.1.1. Nous commencerons par rappeler la construction de la catégorie des motifs purs pour une relation d'équivalence adéquate $\sim$ sur les cycles algébriques. Nous renvoyons à l'ouvrage [1] pour de plus amples détails.

Soient $R$ un anneau et $X$ une $k$-variété projective et lisse sur $k$. Nous notons $A_{\sim}^{r}(X ; R)$ le groupe $R$-linéaire des cycles algébriques de codimension $r$ 
dans $X$ modulo la relation $\sim$. La catégorie $\mathrm{M}_{\sim}(k ; R)$ des motifs purs est définie comme suit. Si $Y$ est une $k$-variété projective et lisse sur $k$, si les $k$-variétés $X_{1}, \ldots, X_{\nu}$ désignent les composantes irréductibles de $X$, l'on note

$$
\operatorname{Corr}_{\sim}^{r}(X, Y ; R)=\bigoplus_{i=1}^{\nu} A_{\sim}^{r+\operatorname{dim}\left(X_{i}\right)}\left(X_{i} \times_{k} Y ; R\right)
$$

le $R$-module des correspondances de degré $r$ modulo $\sim$. Ces groupes sont munis d'une loi de composition associative:

$$
\begin{array}{r}
\operatorname{Corr}_{\sim}^{r}(Y, Z ; R) \otimes_{R} \operatorname{Corr}_{\sim}^{s}(X, Y ; R) \rightarrow \operatorname{Corr}_{\sim}^{r+s}(X, Z ; R) \\
(\beta, \alpha) \mapsto \beta \circ \alpha=p_{X Z *}^{X Y Z}\left(p_{Y Z}^{X Y Z *} \beta \frown p_{X Y}^{X Y Z} \alpha\right) .
\end{array}
$$

L'on construit alors la catégorie $\mathrm{M}_{\sim}(k ; R)$ comme la catégorie dont les objets sont les triplets $(X, p, a)$, où $X$ est une $k$-variété projective et lisse sur $k$, $p \in \operatorname{Corr}^{0}(X, X ; R)$ est un idempotent et $a \in \mathbf{Z}$, et dont les morphismes sont décrits par la formule suivante:

$$
\operatorname{Hom}_{\mathrm{M}_{\sim}(k ; R)}((X, p, a),(Y, q, b))=q \operatorname{Corr}_{\sim}^{b-a}(X, Y ; R) p .
$$

L'on dispose d'un foncteur monoïdal

$$
h_{\sim}: \operatorname{SmProj}_{k}^{\mathrm{op}} \rightarrow \mathrm{M}_{\sim}(k ; R)
$$

qui envoie la $k$-variété $X$, projective et lisse sur $k$, sur le triplet $\left(X, \operatorname{Id}_{X}, 0\right)$, et le morphisme $f: X \rightarrow Y$ entre deux $k$-variétés projectives et lisses sur $k$ sur la correspondance ${ }^{t}\left[\Gamma_{f}\right]$, où $\Gamma_{f}$ désigne le graphe de $f$.

Le $n$-ième twist à la Tate $M(n)$ du motif pur $M=(X, p, a)$ se définit comme le motif $M(n):=(X, p, a+n)$. Il est important de noter que, pour tout motif pur $M$, il existe un entier $n$ et une $k$-variété $X$, projective et lisse sur $k$, tels que $M$ soit un facteur direct de $h_{\sim}(X)(n)$. L'on note 1 le motif $h_{\sim}(\operatorname{Spec}(k))$, et $\mathbf{L}=\mathbf{1}(-1)$ le motif de Lefschetz.

La catégorie $\mathrm{M}_{\sim}(k ; R)$ est une catégorie tensorielle rigide, pseudoabélienne, et l'on a $\operatorname{End}(\mathbf{1})=R$.

4.1.2. Kimura dans [17] et O'Sullivan ont avancé l'idée que le fait que les groupes de cohomologie $H^{*}(X)$, d'une $k$-variété $X$ projective et lisse sur $k$, soient des $K$-espaces vectoriels de dimension finie, pour toute cohomologie de Weil $H^{*}$ (à coefficients dans un corps $K$ ), pourrait se traduire sur son motif de Chow. C'est cette notion correspondant, dans la catégorie des motifs, à la «dimension finie » que nous rappelons maintenant. 
DÉFInItion 4.1. Un motif de Chow $M \in \mathrm{M}_{\text {rat }}(k ; \mathbf{Q})$ est dit pair (resp. impair) s'il existe un entier $n \in \mathbf{N}^{*}$ tel que $\Lambda^{n} M=0$ (resp. $\left.\mathrm{S}^{n} M=0\right)$. Un motif $M \in \mathrm{M}_{\text {rat }}(k ; \mathbf{Q})$ est dit de dimension finie s'il admet une décomposition en somme directe

$$
M \simeq M^{+} \oplus M^{-}
$$

avec $M^{+}$un motif pair et $M^{-}$un motif impair.

En général, il n’y a pas de décomposition canonique en parties paire et impaire des motifs de dimension finie. La sous-catégorie pleine $\mathrm{M}_{\text {rat }}^{\mathrm{fd}}(k ; \mathbf{Q})$ de $\mathrm{M}_{\text {rat }}(k ; \mathbf{Q})$ formée des motifs de dimension finie est une catégorie tensorielle rigide, pseudo-abélienne, qui contient les motifs d'Artin et les motifs des variétés abéliennes. Comme cette sous-catégorie contient également les motifs des $k$-courbes projectives et lisses sur $k$, la formule du blow-up et le théorème de factorisation faible des applications birationnelles assurent que la dimension finie est un invariant birationnel pour les $k$-variétés, projectives et lisses sur $k$, de dimension inférieure ou égale à 3 .

La conjecture de Kimura et O'Sullivan peut maintenant être formulée comme suit. (Nous renvoyons le lecteur à [2, 17, 15] pour de plus amples détails.)

CONJECTURE KS. Tout motif de Chow $M \in \mathrm{M}_{\text {rat }}(k ; \mathbf{Q})$ est de dimension finie.

Soit $\mathrm{M}_{\text {num }}^{\mathrm{fd}}(k ; \mathbf{Q})$ l'image essentielle de $\mathrm{M}_{\text {rat }}^{\mathrm{fd}}(k ; \mathbf{Q})$ dans $\mathrm{M}_{\text {num }}(k ; \mathbf{Q})$. Le théorème de nilpotence, prouvé par Kimura dans [17], implique en particulier que le foncteur

$$
\mathrm{M}_{\mathrm{rat}}^{\mathrm{fd}}(k ; \mathbf{Q}) \rightarrow \mathrm{M}_{\text {num }}^{\mathrm{fd}}(k ; \mathbf{Q})
$$

est conservatif, i.e. jouit de la propriété suivante: un morphisme de la catégorie $\mathrm{M}_{\text {rat }}^{\mathrm{fd}}(k ; \mathbf{Q})$ est un isomorphisme si et seulement si son image dans $\mathrm{M}_{\text {num }}^{\mathrm{fd}}(k ; \mathbf{Q})$ est un isomorphisme.

4.1.3. Dans sa lettre à J.-P. Serre du 16 août 1964, Grothendieck évoque l'existence d'un morphisme d'anneaux

$$
K_{0}\left(\operatorname{Var}_{k}\right) \rightarrow K_{0}\left(\mathrm{M}_{\mathrm{rat}}(k ; \mathbf{Q})\right)
$$


défini par $X \mapsto\left[h_{\text {rat }}(X)\right]$, et que nous appellerons caractéristique d'Euler motivique. Ce morphisme fut initialement ${ }^{7}$ ) construit de manière explicite dans [8], voir également [12, 4].

Remarque 4.2. Soit $k$ un corps de caractéristique zéro. Dans sa lettre à J.-P. Serre, Grothendieck pose la question de savoir si la caractéristique d'Euler motivique est injective. La réponse à cette question est négative. Historiquement, et bien que la réponse n'y figure pas, des contre-exemples peuvent se déduire des travaux de Poonen [29], en considérant un couple de variétés abéliennes isogènes non isomorphes (voir §4.2). Dans [23, Remark 14], d'autres contre-exemples sont obtenus. Plus récemment, Mazza et Weibel ont donné dans [24] une preuve alternative de cette non injectivité.

Nous donnons ici un nouveau type d'exemples d'éléments non triviaux appartenant au noyau de la caractéristique d'Euler motivique. Dans [13], Pedrini et Guletskiǐ ont montré que la classe du motif de la surface de Godeaux complexe $X$, qui est projective et lisse sur $\mathbf{C}$, et de type général, possédait dans l'anneau $K_{0}\left(\mathrm{M}_{\text {rat }}(\mathbf{C} ; \mathbf{Q})\right)$ une décomposition de la forme $\left[h_{\text {rat }}(X)\right]=[\mathbf{L}]^{2} \oplus 9[\mathbf{L}] \oplus[\mathbf{1}]$. Il découle d'un calcul facile que cette décomposition est aussi celle de la surface rationnelle $Y$ obtenue par éclatement de 8 points dans $\mathbf{P}_{\mathbf{C}}^{2}$. Enfin, en vertu de [23, Corollary 1], l'on ne peut avoir $[X]=[Y]$ dans l'anneau $K_{0}\left(\operatorname{Var}_{\mathbf{C}}\right)$. De tels exemples peuvent également être construits à partir des surfaces d'Enriques.

4.1.4. Le groupe de Grothendieck $K_{0}(\mathscr{A})$ d'une catégorie $\mathscr{A}$ abélienne et semi-simple est le groupe abélien libre engendré par les classes d'isomorphisme des objets simples de $\mathscr{A}$. En particulier, deux objets $A$ et $B$ de la catégorie $\mathscr{A}$ sont isomorphes si et seulement si $[A]=[B]$ dans le groupe $K_{0}(\mathscr{A})$.

Cette simple remarque permet de lier, via le foncteur naturel

$$
\mathrm{M}_{\text {rat }}(k ; \mathbf{Q}) \rightarrow \mathrm{M}_{\text {num }}(k ; \mathbf{Q}),
$$

la question PMs à la question KS. Précisément, on a l'énoncé suivant.

Proposition 4.3. Soient $X$ et $Y$ deux $k$-variétés projectives et lisses sur $k$, telles que les motifs de Chow $h_{\text {rat }}(X)$ et $h_{\text {rat }}(Y)$ soient de dimension finie.

(1) Si les $k$-variétés $X$ et $Y$ sont isomorphes par morceaux, alors les motifs de Chow $h_{\text {rat }}(X)$ et $h_{\text {rat }}(Y)$ sont isomorphes dans l'anneau $\mathrm{M}_{\text {rat }}(k ; \mathbf{Q})$.

\footnotetext{
${ }^{7}$ ) A l'heure actuelle, les travaux de Bittner [4] (voir §4.2) ou de Bondarko [6] fournissent deux autres preuves de l'existence de la caractéristique d'Euler motivique.
} 
(2) Si $[X]=[Y]$ dans l'anneau $K_{0}\left(\operatorname{Var}_{k}\right)$, alors les motifs de Chow $h_{\mathrm{rat}}(X)$ et $h_{\text {rat }}(Y)$ sont isomorphes dans l'anneau $\mathrm{M}_{\text {rat }}(k ; \mathbf{Q})$.

(3) En particulier, si la question $\mathrm{KS}$ possède une réponse positive, alors il en va de même pour les questions $\mathrm{PMs}, \mathrm{PCs}, \mathrm{PM}$ et $\mathrm{PC}$.

Cet énoncé découle directement de la proposition suivante.

Proposition 4.4. Soient $M$ et $N$ deux motifs de Chow de dimension finie dans $\mathrm{M}_{\text {rat }}(k ; \mathbf{Q})$. Soient $\bar{M}$ et $\bar{N}$ leurs images dans $\mathrm{M}_{\text {num }}(k ; \mathbf{Q})$. Supposons que $[\bar{M}]=[\bar{N}]$ dans l'anneau $K_{0}\left(\mathrm{M}_{\text {num }}(k ; \mathbf{Q})\right)$. Alors $M$ et $N$ sont isomorphes dans $\mathrm{M}_{\mathrm{rat}}(k ; \mathbf{Q})$.

Démonstration. En vertu du théorème de Jannsen [16], la catégorie $\mathrm{M}_{\text {num }}(k ; \mathbf{Q})$ est semi-simple et abélienne. L'égalité des classes $[\bar{M}]=[\bar{N}]$ dans l'anneau $K_{0}\left(\mathrm{M}_{\text {num }}(k ; \mathbf{Q})\right)$ implique, par la remarque du début du paragraphe 4.1 , que les motifs $\bar{M}$ et $\bar{N}$ sont isomorphes dans $\mathrm{M}_{\text {num }}(k ; \mathbf{Q})$. Par le théorème de nilpotence de Kimura [17], le foncteur

$$
\mathrm{M}_{\text {rat }}^{\mathrm{fd}}(k ; \mathbf{Q}) \rightarrow \mathrm{M}_{\text {num }}^{\mathrm{fd}}(k ; \mathbf{Q})
$$

est conservatif. Donc les motifs $M$ et $N$ sont déjà isomorphes dans $\mathrm{M}_{\mathrm{rat}}(k ; \mathbf{Q})$.

REMARQUE 4.5. Un tel énoncé peut se déduire également des conjectures de Murre-Bloch-Beilinson (voir [9, §2]). La version complète des conjectures de Murre et la conjecture ${ }^{8}$ ) standard D impliquent, à elles deux, la conjecture de Kimura-O'Sullivan (voir [1, 11.5.3.1]). Réciproquement, la dimension finie du motif de Chow $h_{\text {rat }}(X)$ et la conjecture ${ }^{9}$ ) standard $\mathrm{C}(X)$ impliquent que $h_{\text {rat }}(X)$ possède une décomposition de Künneth, pour toute $k$-variété $X$ projective et lisse sur $k$ (ce qui forme une partie des conjectures de Murre, telles qu'énoncées dans [25]).

\subsection{LA QUESTION PM EN PETITES DIMENSIONS}

Soit $k$ un corps algébriquement clos de caractéristique zéro. Comme les motifs de Chow des $k$-courbes, projectives et lisses sur $k$, sont de dimension

\footnotetext{
${ }^{8}$ ) La conjecture standard D prédit que (à torsion près) la relation d'équivalence homologique coïncide avec la relation d'équivalence numérique.

$\left.{ }^{9}\right)$ La conjecture standard $\mathrm{C}(X)$ prédit l'algébricité des projecteurs de Künneth de la $k$-variété $X$. Pour plus de détails sur les conjectures standard (de Grothendieck), voir par exemple le texte de Kleiman [18]
} 
finie dans $\mathrm{M}_{\text {rat }}(k ; \mathbf{Q})$, il découle de la proposition 4.3 que les questions PMs, PM (et donc PCs et PC) admettent des réponses positives pour les $k$-courbes projectives et lisses sur $k$. Nous montrons que les réponses sont encore positives pour les $k$-surfaces, projectives et lisses sur $k$.

PROPOSITION 4.6. Soit $k$ un corps algébriquement clos de caractéristique zéro. Soient $X$ et $Y$ deux $k$-surfaces projectives et lisses sur $k$. Supposons que $[X]=[Y]$ dans l'anneau $K_{0}\left(\operatorname{Var}_{k}\right)$. Alors il existe un isomorphisme de motifs $h_{\text {rat }}(X) \simeq h_{\text {rat }}(Y)$ dans $\mathrm{M}_{\text {rat }}(k ; \mathbf{Q})$.

Démonstration. En vertu de [23, Lemma 9], on sait que les $k$-surfaces $X$ et $Y$ sont birationnellement équivalentes. Comme la dimension finie des motifs est un invariant birationnel des surfaces projectives et lisses, l'on conclut que les motifs de Chow $h_{\text {rat }}(X)$ et $h_{\text {rat }}(Y)$ sont de dimension finie si et seulement si $h_{\text {rat }}(X)$ ou $h_{\text {rat }}(Y)$ est de dimension finie. Nous allons traiter les trois cas suivants découlant de la classification des surfaces.

Si l'une des $k$-surfaces $X$ ou $Y$ est rationnelle, $h_{\text {rat }}(X)$ et $h_{\text {rat }}(Y)$ sont de dimension finie, et donc isomorphes par la proposition 4.3.

$S$ 'il existe une $k$-courbe $C$, connexe, projective et lisse sur $k$, telle que l'une des $k$-surfaces $X$ ou $Y$ est birationnellement équivalente à $\mathbf{P}_{k}^{1} \times{ }_{k} C$, alors il en est de même pour l'autre. Comme le motif de Chow $h_{\text {rat }}(C)$ est de dimension finie, l'on conclut encore que les motifs de Chow $h_{\text {rat }}(X)$ et $h_{\text {rat }}(Y)$ sont de dimension finie, et donc isomorphes par la proposition 4.3.

Supposons enfin que ni la $k$-surface $X$, ni la $k$-surface $Y$ ne soient réglées. Dans ce cas, il existe une $k$-surface $V$, connexe, projective et lisse sur $k$, et deux suites finies

$$
X \stackrel{f}{\rightarrow} V \stackrel{g}{\leftarrow} Y
$$

d'éclatements de points rationnels (i.e. $V$ est le modèle minimal de la classe d'équivalence birationnelle de $X)$. Dans l'anneau $K_{0}\left(\operatorname{Var}_{k}\right)$, la formule de l'éclatement implique qu'il existe deux entiers naturels $m$ et $n$, correspondants aux nombres d'éclatements dans $f$ et $g$ respectivement, tels que:

$$
[X]-m \cdot \mathbf{L}=[V]=[Y]-n \cdot \mathbf{L} .
$$

L'hypothèse entraîne alors que $m=n$ (spécialiser l'égalité par exemple via la caractéristique d'Euler). La formule de l'éclatement pour les motifs de Chow implique le résultat voulu. En effet, l'on déduit l'isomorphisme de motifs de Chow:

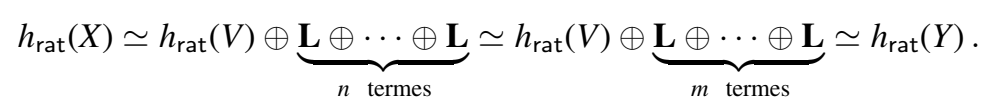


4.2.1. En utilisant le théorème de factorisation faible des applications birationnelles et la résolution des singularités, Bittner a donné dans [4] la description plus simple suivante de l'anneau de Grothendieck des variétés, en termes de générateurs-relations. Soit $k$ un corps algébriquement clos de caractéristique zéro. Posons $K_{0}^{\mathrm{bl}}\left(\operatorname{Var}_{k}\right)$ le groupe abélien obtenu comme le quotient du groupe abélien libre, engendré par les classes d'isomorphismes des $k$-variétés projectives et lisses sur $k$, modulo les relations d'éclatement

$$
[Y]-[E]=[X]-[Z],
$$

où $X$ est une $k$-variété projective et lisse sur $k, Z$ une sous-variété fermée de $X$, lisse sur $k, Y$ l'éclatement de $X$ de centre $Z$ et $E$ le diviseur exceptionnel. Alors le morphisme d'anneaux ${ }^{10}$ ) canonique

$$
K_{0}^{\mathrm{bl}}\left(\operatorname{Var}_{k}\right) \rightarrow K_{0}\left(\operatorname{Var}_{k}\right)
$$

est un isomorphisme.

4.2.2. Soit AV l'ensemble des classes d'isomorphisme de variétés abéliennes sur le corps $k$ supposé algébriquement clos de caractéristique zéro (qui est un monoïde pour le produit fibré au-dessus de $k$ ). L'on peut alors définir un morphisme d'anneaux

$$
K_{0}\left(\operatorname{Var}_{k}\right) \rightarrow \mathbf{Z}[\mathrm{AV}]
$$

par additivité en imposant que la classe d'une $k$-variété $X$, projective et lisse sur $k$, soit envoyée sur sa variété d'Albanese $\mathrm{Alb}(X)$ (cf. [29] ou [23]). En particulier, l'on déduit de l'existence de ce morphisme que deux $k$-variétés, projectives et lisses sur $k$, telles que $[X]=[Y]$ dans l'anneau $K_{0}\left(\operatorname{Var}_{k}\right)$ ont des variétés d'Albanese isomorphes (et, par dualité, des variétés de Picard isomorphes). En outre, en tant que groupes abstraits, $\operatorname{Pic}^{0}(X)$ et $\operatorname{Pic}^{0}(Y)$ sont isomorphes.

4.2.3. La présentation de l'anneau $K_{0}\left(\operatorname{Var}_{k}\right)$, via les relations d'éclatement, et les théorèmes de finitude classiques permettent d'énoncer les résultats suivants.

Proposition 4.7. Soit $k$ un corps de caractéristique zéro. Soient $X$ et $Y$ deux $k$-variétés connexes, projectives et lisses sur $k$, telles que $[X]=[Y]$ dans l'anneau $K_{0}\left(\operatorname{Var}_{k}\right)$.

$\left.{ }^{10}\right)$ Le produit est ici encore induit par le produit fibré au-dessus de $k$. 
(1) Les groupes de Néron-Severi $\mathrm{NS}(X)$ et $\mathrm{NS}(Y)$ sont isomorphes.

(2) Si le corps $k$ est une extension de type fini de $\mathbf{Q}$, alors les groupes de Picard $\operatorname{Pic}(X)$ et $\operatorname{Pic}(Y)$ sont isomorphes.

Démonstration. Soit $\mathscr{A}$ la catégorie des groupes abéliens de type fini. C'est une catégorie additive. Nous considérons son groupe de Grothendieck $K_{0}(\mathscr{A})$. L'on montre d'abord le second point.

(2) Par hypothèse, il découle de [14, Proposition 6.1] que le groupe de Picard d'une $k$-variété, projective et lisse sur $k$, est de type fini. Il définit donc un objet de $\mathscr{A}$. Ainsi l'on construit un morphisme de groupes abéliens

$$
\text { Pic: } \mathbf{Z}\left[\operatorname{SmPr}_{k}\right] \rightarrow \mathbf{Z}[\mathscr{A}]
$$

qui à la classe d'isomorphisme d'une $k$-variété $V$, projective et lisse sur $k$, associe la classe d'isomorphisme de son groupe de $\operatorname{Picard}\{\operatorname{Pic}(V)\}$. Comme, pour toute $k$-variété $V$, projective et lisse sur $k$, l'on a:

$$
\operatorname{Pic}(V)=\mathrm{CH}^{1}(V)=\operatorname{Hom}_{\mathrm{M}_{\text {rat }}(k ; \mathbf{Z})}\left(\mathbf{L}, h_{\text {rat }}(V)\right),
$$

la formule de l'éclatement pour les motifs de Chow assure, via [4], que le morphisme Pic induit un morphisme de groupes

$$
\text { Pic: } K_{0}\left(\operatorname{Var}_{k}\right) \rightarrow K_{0}(\mathscr{A}) \text {. }
$$

Il découle du théorème de structure des groupes abéliens de type fini que deux objets $A$ et $B$ de $\mathscr{A}$ sont isomorphes si et seulement si $[A]=[B]$ dans l'anneau $K_{0}(\mathscr{A})$. Le résultat se déduit alors facilement.

(1) La preuve est similaire, en utilisant cette fois les groupes de NéronSeveri. Si $V$ est une $k$-variété, projective et lisse sur $k$, son groupe de Néron-Severi, qui est un groupe abélien de type fini, est donné par la formule:

$$
\mathrm{NS}(V)=\operatorname{Hom}_{\mathrm{M}_{\mathrm{alg}}(k ; \mathbf{Z})}\left(\mathbf{L}, h_{\mathrm{alg}}(V)\right) .
$$

Comme précédemment, la formule de l'éclatement pour les motifs de Chow permet de construire, via [4], un morphisme de groupes

$$
\mathrm{NS}: K_{0}\left(\operatorname{Var}_{k}\right) \rightarrow K_{0}(\mathscr{A})
$$

qui associe, à la classe d'une $k$-variété, projective et lisse sur $k$, la classe de son groupe de Néron-Severi.

REMARQue 4.8. Soit $k$ un corps algébriquement clos de caractéristique zéro. Si $Z$ est une $k$-variété, projective et lisse sur $k$, son groupe de NéronSeveri est donné par la formule:

$$
\operatorname{NS}(Z)=\operatorname{Pic}(Z) / \operatorname{Pic}^{0}(Z)
$$


En vertu de la proposition 4.7, l'on déduit que si deux $k$-variétés $X$ et $Y$, projectives et lisses sur $k$, ont même classe dans l'anneau de Grothendieck des variétés, alors les groupes $\operatorname{Pic}(X)_{\mathbf{Q}}$ and $\operatorname{Pic}(Y)_{\mathbf{Q}}$ sont isomorphes. L'on conclut donc que la question $P C s^{1}$ possède une réponse positive. Si $n=\operatorname{dim}(X)$, les questions $\mathrm{PC}^{n}$ et $P C s^{n}$ ont également une réponse positive, car les groupes de Chow de codimension $n$ sont des invariants birationnels (cf. par exemple $[23$, Corollary 6]).

\section{GÉOMÉTRIE ALGÉBRIQUe PAR MORCEAUX ET QUESTION KP}

Dans cette section, nous nous intéressons à la question KP. Le théorème 5.4, la proposition 5.5 ainsi que la remarque 5.7 fournissent des éléments de réponse originaux, en direction d'une réponse positive à cette question. Nos énoncés complètent ceux donnés antérieurement dans [32], mais demeurent partiels.

5.1. Soit $k$ un corps algébriquement clos de caractéristique zéro. Soient $X$ et $Y$ deux $k$-variétés connexes, propres et lisses sur $k$, et $K$-équivalentes. Il sera commode de disposer de la notion de lieu $K$-exceptionnel que nous introduisons maintenant.

Par définition, il existe une $k$-variété $Z$, connexe, propre et lisse sur $k$, et deux morphismes birationnels de $k$-schémas $X \stackrel{f}{\leftarrow} Z \stackrel{g}{\rightarrow} Y$ tels que $K_{Z / X}=K_{Z / Y}$. Il existe donc trois ouverts $U, V$ et $W$ de $X, Y$ et $Z$ respectivement tels que les morphismes $f$ et $g$ induisent par restriction des isomorphismes de $k$-schémas $U \stackrel{f}{\leftarrow} W \stackrel{g}{\rightarrow} V$. Le morphisme de $k$-schémas $h:=g_{\mid W} \circ\left(f_{\mid W}\right)^{-1}$ produit donc un isomorphisme de $k$-schémas $U \rightarrow V$. Désignons par $C_{X}$ et $C_{Y}$ les uniques sous-variétés fermées et réduites de $X$ et $Y$ respectivement, dont les supports sont égaux aux ensembles $X \backslash U$ et $Y \backslash V$. La donnée d'un tel couple $\left(C_{X}, C_{Y}\right)$ constitue ce que nous appellerons un lieu $K$-exceptionnel de $X$ et $Y$ dans la suite. Une telle donnée ne peut être unique. Remarquons cependant que, si $\left(C_{X}, C_{Y}\right)$ est un lieu $K$-exceptionnel d'une paire de $k$-variétés $X, Y$ supposées $K$-équivalentes, alors, en vertu du corollaire 1.3, la dimension de $C_{X}$ est égale à celle de $C_{Y}$. 
5.2. Ci-après, nous établissons un lemme que nous utiliserons par la suite.

LEMME 5.1. Soit $k$ un corps algébriquement clos de caractéristique zéro. Soient $V$ et $W$ deux $k$-variétés réduites de même dimension $d$. Si $d \leqslant 1$, si $\mathrm{SB}(V)=\mathrm{SB}(W)$ et si les $k$-variétés $V$ et $W$ ont le même nombre de composantes irréductibles de dimension $d$, alors les $k$-variétés $V$ et $W$ sont isomorphes par morceaux.

Démonstration. L'unique difficulté réside en dimension 1. Par hypothèse, il existe un élément $\alpha \in K_{0}\left(\operatorname{Var}_{k}\right)$ tel que $[V]-[W]=\alpha \cdot \mathbf{L}$ dans l'anneau $K_{0}\left(\operatorname{Var}_{k}\right)$. Soit $n$ le nombre de composantes irréductibles de $V$ de dimension 1. Pour tout $i, 1 \leqslant i \leqslant n$, nous désignons par $V_{i}$ et $W_{i}$ respectivement les modèles projectifs et lisses sur $k$ de chacune des composantes irréductibles de dimension 1 de $V$ et $W$. Il existe alors un entier $m \in \mathbf{Z}$ tel que

$$
\sum_{i=1}^{n}\left[V_{i}\right]-\sum_{j=1}^{n}\left[W_{j}\right]=\alpha \cdot \mathbf{L}+m,
$$

dans l'anneau $K_{0}\left(\operatorname{Var}_{k}\right)$. Si l'on applique le morphisme SB à cette relation, on peut conclure que $m=0$, puis, quitte à renuméroter, que $\mathrm{SB}\left(V_{i}\right)=\mathrm{SB}\left(W_{i}\right)$, pour tout $i, 1 \leqslant i \leqslant n$. Autrement dit, pour tout $i, 1 \leqslant i \leqslant n$, les $k$-courbes $V_{i}$ et $W_{i}$ sont isomorphes. L'on conclut donc que les composantes irréductibles de dimension 1 de $V$ et $W$ correspondantes sont birationnellement équivalentes. Il existe donc des sous-variétés fermées et réduites $C_{V}$ et $C_{W}$ de $V$ et $W$ respectivement, de dimension 0 , telles que

$$
\left[C_{V}\right]-\left[C_{W}\right]=\alpha \cdot \mathbf{L} .
$$

dans l'anneau $K_{0}\left(\operatorname{Var}_{k}\right)$. Par conséquent, $\mathrm{SB}\left(C_{V}\right)=\mathrm{SB}\left(C_{W}\right)$. Comme $C_{V}$ et $C_{W}$ sont des sommes disjointes de points rationnels, cette dernière relation implique que leurs ensembles sous-jacents ont le même cardinal, ou que $C_{V}$ et $C_{W}$ sont isomorphes. Par conséquent, les $k$-variétés $V$ et $W$ sont isomorphes par morceaux.

5.3. Soit $f: X \rightarrow Y$ une application birationnelle entre deux $k$-variétés connexes, propres et lisses sur $k$, de dimension $d$. Notons $C_{X}$ le lieu exceptionnel de $f$ (i.e. l'ensemble des points de $X$ où $f$ n'est pas un isomorphisme local). Notons $U_{X}$ l'ouvert de $X$ défini par $X \backslash C_{X}$. Comme $f$ est supposée birationnelle, il découle du théorème principal de Zariski l'existence d'un ouvert $V_{Y}$ de $Y$ tel que $f$ induise un isomorphisme de $k$-schémas $U_{X} \rightarrow V_{Y}$. Nous notons $C_{Y}$ l'unique sous-variété fermée et réduite de $Y$ dont le support est égal à l'ensemble $Y \backslash V_{Y}$. L'énoncé suivant est une conséquence du lemme 5.1. 
LEMME 5.2. Soit $k$ un corps algébriquement clos de caractéristique zéro. Soit $f: X \rightarrow Y$ une application birationnelle entre deux $k$-variétés connexes, propres et lisses sur $k$, de dimension $d \geqslant 3$. Notons $C_{X}$ le lieu exceptionnel de $f$. Supposons que $1 \geqslant \operatorname{dim}\left(C_{X}\right) \geqslant \operatorname{dim}\left(C_{Y}\right)$, et que les nombres de Betti $b_{2}(X)$ et $b_{2}(Y)$ sont égaux. Alors les $k$-variétés sont isomorphes par morceaux.

Démonstration. Par hypothèse, l'on a dans l'anneau $K_{0}\left(\operatorname{Var}_{k}\right)$ la relation suivante

$$
[X]-\left[C_{X}\right]=[Y]-\left[C_{Y}\right] .
$$

Si l'on applique le morphisme $\mathrm{SB}$ à cette relation (5.1), l'on déduit que $\mathrm{SB}\left(C_{X}\right)=\mathrm{SB}\left(C_{Y}\right)$, puisque les $k$-variétés $X$ et $Y$ sont supposées propres et lisses sur $k$, et birationnellement équivalentes. Si l'on applique le morphisme $P$ à la relation (5.1), l'on obtient une relation de la forme suivante dans l'anneau $\mathbf{Z}[u]$, avec $a_{i}, a_{i}^{\prime} \in \mathbf{Z}$, pour tout $i, 0 \leqslant i \leqslant 2$ :

$$
\sum_{i=0}^{2 d} b_{i}(X) u^{i}-\left(a_{2} u^{2}+a_{1} u+a_{0}\right)=\sum_{i=0}^{2 d} b_{i}(Y) u^{i}-\left(a_{2}^{\prime} u^{2}+a_{1}^{\prime} u+a_{0}^{\prime}\right) .
$$

Comme $b_{2}(X)=b_{2}(Y)$, l'on conclut que $a_{2}=a_{2}^{\prime}$. Il découle alors de l'hypothèse et de la remarque précédente que la dimension de $C_{X}$ est égale à celle de $C_{Y}$. Si $\operatorname{dim}\left(C_{X}\right)=1$, les $k$-variétés $C_{X}$ et $C_{Y}$ ont en outre le même nombre de composantes irréductibles de dimension 1. Le lemme 5.1 permet alors de conclure. $\operatorname{Si} \operatorname{dim}\left(C_{X}\right)=0$, l'on conclut directement grâce à la relation $\mathrm{SB}\left(C_{X}\right)=\mathrm{SB}\left(C_{Y}\right)$.

REMARQUE 5.3. Les arguments utilisés dans la preuve du lemme 5.2 permettent d'obtenir certains cas particuliers quand $\operatorname{dim}\left(C_{X}\right) \geqslant 2$. En voici une illustration. Soient $X$ et $Y$ deux $k$-variétés $K$-équivalentes. Fixons $\left(C_{X}, C_{Y}\right)$ un lieu $K$-exceptionnel. Supposons que les $k$-variétés $C_{X}$ et $C_{Y}$ soient des $k$-surfaces, projectives et lisses sur $k$. Alors les $k$-variétés $X$ et $Y$ sont isomorphes par morceaux.

Le lemme 5.2 et la remarque 5.3 précédents soulignent le fait que la réponse à la question $\mathrm{KP}$ s'obtient facilement en dimension $d \leqslant 3$, mais que la question sous-jacente reste toutefois pertinente en dimension supérieure.

5.4. Rappelons le résultat suivant, que l'on peut déduire de [32, Proposition 3.2, Lemme 3.4]. 
THÉORÈME 5.4. Soit $k$ un corps algébriquement clos de caractéristique zéro. Soient $X$ et $Y$ deux $k$-variétés $K$-équivalentes. Fixons un lieu $K$-exceptionnel $\left(C_{X}, C_{Y}\right)$. Notons $C_{*}^{\max }$ l'ensemble des points de $C_{*}$ de dimension maximale. Alors il existe une bijection

$$
\sigma: C_{X}^{\max } \rightarrow C_{Y}^{\max },
$$

telle que, pour tout $x \in C_{X}^{\max }$, il existe un entier $s \in \mathbf{N}$ et un isomorphisme de k-algèbres $\varphi: \kappa(x)\left(\mathrm{T}_{1}, \ldots, \mathrm{T}_{s}\right) \rightarrow \kappa(\sigma(x))\left(\mathrm{T}_{1}, \ldots, \mathrm{T}_{s}\right)$.

La preuve de cet énoncé utilise, de manière cruciale le théorème de Kontsevich, sous la forme du corollaire 1.3, et des résultats techniques provenant de la théorie géométrique de l'intégration motivique.

5.5. Le théorème 5.4 souligne l'importance du problème de simplification «à la Zariski»dans les questions au centre de ce travail. Dans certains cas particuliers, le théorème 5.4 permet malgré tout de répondre à la question KP . Par exemple, nous pouvons déduire l'énoncé suivant.

PROPOSITION 5.5. Soit $k$ un corps algébriquement clos de caractéristique zéro. Soient $X$ et $Y$ deux $k$-variétés connexes, projectives et lisses sur $k$, $K$-équivalentes, de dimension $d$. Fixons $\left(C_{X}, C_{Y}\right)$ un lieu $K$-exceptionnel. Supposons

a) ou bien $\operatorname{dim}\left(C_{X}\right) \leqslant 2$;

b) ou bien que les $k$-variétés $C_{X}$ et $C_{Y}$ sont isomorphes à des sommes disjointes de $k$-variétés de la forme $S \times_{k} \mathbf{P}_{k}^{r-2}(r \geqslant 3)$, où $S$ est une $k$-surface intègre projective.

Alors les $k$-variétés $X$ et $Y$ sont isomorphes par morceaux.

Démonstration. Par hypothèse, on a la relation

$$
[Y]-\left[C_{Y}\right]=[X]-\left[C_{X}\right]
$$

dans l'anneau $K_{0}\left(\operatorname{Var}_{k}\right)$. En vertu du corollaire 1.3, l'on peut conclure que les $k$-variétés $C_{X}$ et $C_{Y}$ ont la même dimension, et le même nombre de composantes irréductibles de dimension maximale. Notons $r$ cette dimension, et $f_{0}: X \backslash C_{X} \rightarrow Y \backslash C_{Y}$ l'isomorphisme de $k$-schémas.

Supposons que l'hypothèse faite au point a) soit valide. Ce cas a été traité dans [32, Théorème 3.5]. Nous en redonnons ici la preuve. Grâce au théorème 5.4 , l'on construit deux sous-variétés fermées et réduites $D_{X}$ et $D_{Y}$ de $C_{X}$ et $C_{Y}$ respectivement, de dimension au plus 1 , et un isomorphisme de 
$k$-schémas $f_{1}: C_{X} \backslash D_{X} \rightarrow C_{Y} \backslash D_{Y}$. En particulier, cet isomorphisme permet de réécrire la relation (5.3) sous la forme

$$
[X]-\left[D_{X}\right]=[Y]-\left[D_{Y}\right],
$$

dans l'anneau $K_{0}\left(\operatorname{Var}_{k}\right)$. Si l'on applique le morphisme $\mathrm{SB}$ à cette relation, l'on conclut que $\mathrm{SB}\left(D_{X}\right)=\mathrm{SB}\left(D_{Y}\right)$ dans l'anneau $\mathbf{Z}[S B]$. Par ailleurs, le corollaire 1.3 permet encore d'affirmer que les $k$-variétés $D_{X}$ et $D_{Y}$ ont la même dimension et le même nombre de composantes irréductibles de dimension maximale. L'on déduit alors du lemme 5.1 que les $k$-variétés $D_{X}$ et $D_{Y}$ sont isomorphes par morceaux via $\left(f_{2}, f_{3}\right)$. La donnée de $\left(f_{0}, f_{1}, f_{2}, f_{3}\right)$ définit un isomorphisme par morceaux entre $X$ et $Y$.

Supposons que l'hypothèse faite au point $\mathrm{b}$ ) soit valide. Soit $C$ une composante irréductible de dimension maximale de $C_{X}$. Posons alors $C^{\prime}:=\sigma(C)$, où $\sigma$ est la bijection construite dans le théorème 5.4. Par hypothèse, il existe des $k$-surfaces intègres $S_{X}$, et $S_{Y}$, telles que la $k$-variété $C$ (resp. $C^{\prime}$ ) est isomorphe à $S_{X} \times{ }_{k} \mathbf{P}_{k}^{s}$ (resp. $S_{Y} \times{ }_{k} \mathbf{P}_{k}^{s}$ ), avec $s=r-2$. L'on peut déduire du théorème 5.4 que $S$ et $S^{\prime}$ sont stablement birationnelles, donc birationnellement équivalentes en vertu du théorème de Castelnuovo. Il existe donc des sous-variétés fermées et réduites $F_{X}$ et $F_{Y}$ de $S_{X}$ et $S_{Y}$ respectivement, de dimension au plus 1 , telles que $S_{X} \backslash F_{X} \cong S_{Y} \backslash F_{Y}$.

Supposons que la $k$-variété $C_{X}$ (resp. $C_{Y}$ ) possède $n$ composantes irréductibles de dimension maximale. Soient $S_{X}^{1}, \ldots, S_{X}^{n}$ (resp. $S_{Y}^{1}, \ldots, S_{Y}^{n}$ ) des $k$-surfaces intègres telles que les composantes irréductibles $C_{1}, \ldots, C_{n}$ (resp. $C_{1}^{\prime}, \ldots, C_{n}^{\prime}$ ) de dimension maximale de $C_{X}$ (resp. $C_{Y}$ ) soient birationnellement équivalentes à $S_{X}^{1} \times_{k} \mathbf{P}_{k}^{s}, \ldots, S_{X}^{n} \times_{k} \mathbf{P}_{k}^{s}\left(\right.$ resp. $\left.S_{Y}^{1} \times_{k} \mathbf{P}_{k}^{s}, \ldots, S_{Y}^{n} \times_{k} \mathbf{P}_{k}^{s}\right)$. Quitte à renuméroter, et en vertu de la remarque précédente, nous construisons des sous-variétés fermées et réduites $F_{X}^{1}, \ldots, F_{X}^{n}$ (resp. $F_{Y}^{1}, \ldots, F_{Y}^{n}$ ) respectivement de $S_{X}^{1}, \ldots, S_{X}^{n}$ (resp. $S_{Y}^{1}, \ldots, S_{Y}^{n}$ ), de dimension au plus 1, telles que, pour tout $i, 1 \leqslant i \leqslant n, S_{X}^{i} \backslash F_{X}^{i} \cong S_{Y}^{i} \backslash F_{Y}^{i}$. Les relations de découpage dans l'anneau $K_{0}\left(\operatorname{Var}_{k}\right)$ permettent donc d'écrire

$$
[X]-\left[\mathbf{P}_{k}^{s}\right]\left[F_{X}\right]=[Y]-\left[\mathbf{P}_{k}^{s}\right]\left[F_{Y}\right],
$$

avec $F_{X}:=\sqcup_{i=1}^{n} F_{X}^{i}$ et $F_{Y}=\sqcup_{i=1}^{n} F_{Y}^{i}$. Notons que nous avons construit un isomorphisme de $k$-schémas $f_{1}: C_{X} \backslash\left(F_{X} \times_{k} \mathbf{P}_{k}^{s}\right) \rightarrow C_{Y} \backslash\left(F_{Y} \times_{k} \mathbf{P}_{k}^{s}\right)$.

Comme $\left[\mathbf{P}_{k}^{s}\right]$ est un élément inversible de l'anneau $\widehat{\mathcal{M}}_{k}$, l'on conclut par le corollaire 1.3 que les $k$-variétés $F_{X}$ et $F_{Y}$ ont la même dimension et le même nombre de composantes irréductibles de dimension maximale. En outre, en appliquant le morphisme SB à la relation (5.4), l'on obtient que $\mathrm{SB}\left(F_{X}\right)=\mathrm{SB}\left(F_{Y}\right)$. L'on déduit du lemme 5.11'existence d'un isomorphisme 
par morceaux entre $F_{X}$ et $F_{Y}$, ce qui induit naturellement l'existence d'un isomorphisme par morceaux $\left(f_{2}, f_{3}\right)$ entre $F_{X} \times{ }_{k} \mathbf{P}_{k}^{s}$ et $F_{Y} \times{ }_{k} \mathbf{P}_{k}^{s}$. La donnée de $\left(f_{0}, f_{1}, f_{2}, f_{3}\right)$ définit alors un isomorphisme par morceaux entre $X$ et $Y$.

COROLlaIRE 5.6. Soit $k$ un corps algébriquement clos de caractéristique zéro.

(1) La réponse à la question $\mathrm{KP}$ est positive pour tout couple de $k$-variétés de Calabi-Yau $(X, Y)$, de dimension au plus 4, birationnellement équivalentes. En particulier, deux $k$-variétés de Calabi-Yau $X$ et $Y$, de dimension au plus 4, sont isomorphes par morceaux si et seulement si elles sont birationnellement équivalentes.

(2) Si la conjecture KS est valide pour les $k$-variétés de Calabi-Yau de dimension au plus 4. Alors les réponses aux questions $\mathrm{KM}, \mathrm{KC}$ sont positives pour tout couple de $k$-variétés de Calabi-Yau $(X, Y)$, birationnellement équivalentes, de dimension au plus 4.

Démonstration. La première assertion provient du fait que l'on peut choisir un lieu $K$-exceptionnel $\left(C_{X}, C_{Y}\right)$ tel que les $k$-variétés $C_{X}$ et $C_{Y}$ soient de codimension au moins 2 (cf. [3, Proposition 3.1]), et de la proposition 5.5. La seconde assertion est claire.

REMARQUE 5.7. Soit $k$ un corps algébriquement clos de caractéristique zéro. Soient $X$ et $Y$ deux $k$-variétés de Calabi-Yau, de même dimension $d$, telles que $[X]=[Y]$ dans l'anneau $K_{0}\left(\operatorname{Var}_{k}\right)$. En vertu de [23, Theorem 2], l'on conclut que les $k$-variétés $X$ et $Y$ sont birationnellement équivalentes. Si $d \leqslant 4$, le corollaire 5.6 assure qu'elles sont en fait isomorphes par morceaux. La question LL admet donc une réponse positive pour tout couple de $k$-variétés de Calabi-Yau, de dimension au plus 4.

\section{BIBLIOGRAPHIE}

[1] ANDRÉ, Y. Une introduction aux motifs (motifs purs, motifs mixtes, périodes). Panoramas et Synthèses 17. Société Mathématique de France, Paris, 2004.

[2] — Motifs de dimension finie (d'après S.-I. Kimura, P. O'Sullivan....). Séminaire Bourbaki. Vol. 2003/2004, Exp. No. 929. Astérisque 299, 2005.

[3] BatyreV, V. V. Birational Calabi-Yau $n$-folds have equal Betti numbers. In: New Trends in Algebraic Geometry (Warwick, 1996), 1-11. London Mathematical Society Lecture Note Series 264. Cambridge Univ. Press, Cambridge, 1999. 
[4] BitTNER, F. The universal Euler characteristic for varieties of characteristic zero. Compos. Math. 140 (2004), 1011-1032.

[5] BLICKLE, M. A short course on geometric motivic integration. In: Motivic Integration and its Interactions with Model Theory and Non-Archimedean Geometry, Volume I, 189-243. R. Cluckers, J. Nicaise and J. Sebag (editors). London Mathematical Society Lecture Note Series 383. Cambridge Univ. Press, Cambridge, 2011.

[6] Bondarko, M. V. Differential graded motives: weight complex, weight filtrations and spectral sequences for realizations; Voevodsky versus Hanamura. J. Inst. Math. Jussieu 8 (2009), 39-97.

[7] FU, B. and C.-L. WANG. Motivic and quantum invariance under stratified Mukai flops. J. Differential Geom. 80 (2008), 261-280.

[8] Gillet, H. and C. Soulé. Descent, motives and $K$-theory. J. Reine Angew. Math. 478 (1996), 127-176.

[9] GötTsCHE, L. On the motive of the Hilbert scheme of points on a surface. Math. Res. Lett. 8 (2001), 613-627.

[10] Gromov, M. Endomorphisms of symbolic algebraic varieties. J. Eur. Math Soc. 1 (1999), 109-197.

[11] GrothENDIECK, A. et J. A. DieudonnÉ. Éléments de Géométrie Algébrique I. Grundlehren der Mathematischen Wissenschaften 166. Springer-Verlag, Berlin, 1971.

[12] GuilléN, F. et V. NAVARro AZnAR. Un critère d'extension des foncteurs définis sur les schémas lisses. Publ. Math. Inst. Hautes Études Sci. 95 (2002), $1-91$.

[13] GuletskiĬ, V. and C. PedrinI. The Chow motive of the Godeaux surface. In: Algebraic Geometry, 179-195. de Gruyter, Berlin, 2002.

[14] GuRAlnick, R., D. B. JafFe, W. RASKind and R. Wiegand. On the Picard group: torsion and the kernel induced by a faithfully flat map. J. Algebra 183 (1996), 420-455.

[15] IVORRA, F. Finite dimensional motives and applications (following S.-I. Kimura, P. O'Sullivan and others). In: Autour des motifs-École d'été FrancoAsiatique de Géométrie Algébrique et de Théorie des Nombres, Volume II. Panoramas et Synthèses, to appear.

[16] JANnsEn, U. Motives, numerical equivalence, and semi-simplicity. Invent. Math. 107 (1992), 447-452.

[17] KimURA, S.-I. Chow groups are finite dimensional, in some sense. Math. Ann. 331 (2005), 173-201.

[18] Kleiman, S. L. The standard conjectures. In: Motives (Seattle, WA, 1991), 3-20. Proceedings of Symposia in Pure Mathematics 55, Part 1. Amer. Math. Soc., Providence, RI, 1994.

[19] Kontsevich, M. Exposé à l'Université Paris 11 (Orsay). 7 décembre 1995. (Non publié.)

[20] Lamy, S. and Sebag, J. Birational self-maps and piecewise algebraic geometry. Preprint arXiv: 1112.5706v1 (2011). To appear in Journal of Mathematical Sciences, the University of Tokyo.

[21] LARSEN, M. and V. A. LuNTS. Motivic measures and stable birational geometry. Mosc. Math. J. 3 (2003), 85-95, 259. 
[22] LEE, Y.-P., H.-W. LIN and C.-L. WANG. Flops, motives, and invariance of quantum rings. Ann. of Math. (2) 172 (2010), 243-290.

[23] LiU, Q. and J. Sebag. The Grothendieck ring of varieties and piecewise isomorphisms. Math. Z. 265 (2010), 321-342.

[24] MaZzA, C. and C. Weibel. Schur finiteness in $\lambda$-rings. Preprint arXiv: 1011.1444v2 (2010-11).

[25] MurRe, J.P. On a conjectural filtration on the Chow groups of an algebraic variety. I. The general conjectures and some examples. Indag. Math. (N.S.) 4 (1993), 177-188.

[26] Olivier, J.-P. Anneaux absolument plats universels et épimorphismes à but réduits. Séminaire Samuel 2 (1967-1968), Exposé no. 6. Sécrétariat Mathématique, Paris.

[27] Le foncteur $T^{-\infty}$. Globalisation du foncteur $T$. Séminaire Samuel 2 (1967-1968), Exposé no. 9. Sécrétariat Mathématique, Paris.

[28] ORLov, D. O. Derived categories of coherent sheaves, and motives. Uspekhi Mat. Nauk 60 (2005), 231-232; translation in Russian Math. Surveys 60 (2005), 1242-1244.

[29] Poonen, B. The Grothendieck ring of varieties is not a domain. Math. Res. Lett. 9 (2002), 493-497.

[30] Sebag, J. Intégration motivique sur les schémas formels. Bull. Soc. Math. France 132 (2004), 1-54.

[31] - Variations on a question of Larsen and Lunts. Proc. Amer. Math. Soc. 138 (2010), 1231-1242.

[32] - Variétés $K$-équivalentes et géométrie par morceaux. Arch. Math. (Basel) 94 (2010), 207-217.

[33] VEYs, W. Arc spaces, motivic integration and stringy invariants. In: Singularity Theory and its Applications, 529-572. Advanced Studies in Pure Mathematics 43. Math. Soc. Japan, Tokyo, 2006.

[34] WANG, C.-L. $K$-equivalence in birational geometry. In: Second International Congress of Chinese Mathematicians, 199-216. New Studies in Advanced Mathematics 4. Int. Press, Somerville, MA, 2004.

(Reçu le 22 septembre 2010; version révisée reçue le 16 février 2012)

Florian Ivorra

Julien Sebag

Institut de recherche mathématique de Rennes

UMR 6625 du CNRS

Université de Rennes 1

Campus de Beaulieu

35042 Rennes cedex (France)

e-mail: florian.ivorra@univ-rennes1.fr, julien.sebag@univ-rennes1.fr 